\title{
Effect of Meloidogyne incognita Inoculum Density and Application Rate of Paecilomyces lilacinus Strain 251 on Biocontrol Efficacy and Colonization of Egg Masses Analyzed by Real-Time Quantitative PCR
}

\author{
S. Kiewnick, S. Neumann, R. A. Sikora, and J. E. Frey
}

First and fourth authors: Research Station Agroscope Changins-Waedenswil ACW, Plant Protection and Extension Fruit and Vegetables. Schloss P.O. Box, 8820 Waedenswil, Switzerland; and second and third authors: Institute for Crop Science and Resource Conservation, INRES, University of Bonn, Nussallee 9, D-53115 Bonn, Germany.

Accepted for publication 25 August 2010.

\begin{abstract}
Kiewnick, S., Neumann, S., Sikora, R. A., and Frey, J. E. 2011. Effect of Meloidogyne incognita inoculum density and application rate of Paecilomyces lilacinus strain 251 on biocontrol efficacy and colonization of egg masses analyzed by real-time quantitative PCR. Phytopathology 101:105-112.

The fungal biocontrol agent, Paecilomyces lilacinus strain 251 (PL251), was evaluated for its potential to control the root-knot nematode Meloidogyne incognita on tomato at varying application rates and inoculum densities. Conversely to previous studies, significant doseresponse relationships could not be established. However, we demon-

sufficient to reduce root galling by $45 \%$ and number of egg masses by $69 \%$ when averaged over inoculum densities of 100 to 1,600 eggs and infective juveniles per $100 \mathrm{~cm}^{3}$ of soil. To determine the role of colonization of M. incognita egg masses by PL251 for biocontrol efficacy, a real-time quantitative polymerase chain reaction (PCR) assay with a detection limit of 10 CFU/egg mass was used. Real-time PCR revealed a significant relationship between egg mass colonization by PL251 and the dose of product applied to soil but no correlation was found between fungal density and biocontrol efficacy or nematode inoculum level. These results demonstrate that rhizosphere competence is not the key mode of action for PL251 in controlling M. incognita on tomato.
\end{abstract} strated that a preplanting soil treatment with the lowest dose of commercially formulated PL251 $\left(2 \times 10^{5} \mathrm{CFU} / \mathrm{g}\right.$ soil $)$ was already
Additional keywords: egg-pathogenic fungus, plant-parasitic nematodes.
Biocontrol of root-knot nematodes. Root-knot nematodes (RKN) (Meloidogyne spp.) cause $\approx 5 \%$ of the worldwide economic crop losses. Particularly in tropical and subtropical agriculture, RKN are causing dramatic yield losses on vegetables (16). Environmental side effects and human health risks associated with chemical control combined with increasing governmental regulation have led to more interest in nematode control alternatives (2). However, biocontrol agents are often not thought of as acceptable alternatives to chemical nematicides. Nematophagous fungi often show a biocontrol efficacy which lacks broad-spectrum activity, with sometimes inconsistent performance. In addition, biocontrol efficacy is defined by a slower action (sometimes incomplete) when compared with chemicals (33). Therefore, only a few products have been commercialized thus far (24). Based on the nematophagous fungus Pochonia chlamydosporia var. catenulata (synonym: Verticillium chlamydosporium), a management system for control of RKN in organic vegetable production in Cuba has been developed by Kerry and HidalgoDiaz (19). Bacillus firmus, commercially available as Bionem, has also shown promise for control of RKN (2).

The egg-pathogenic fungus Paecilomyces lilacinus (Thom) Samson is one of the most widely tested soil Hyphomycetes for biological control of plant-parasitic nematodes (4). The commercial P. lilacinus strain 251 (PL251) has been registered with the United States Environmental Protection Agency as a biological nematicide under the trade name MeloCon WG and was

Corresponding author: S. Kiewnick;

E-mail address: sebastian.kiewnick@acw.admin.ch

doi:10.1094/PHYTO-03-10-0090

(C) 2011 The American Phytopathological Society recently included as active substance into Annex I of the European Directive 91/414 EEC $(3,27)$. The potential of PL251 to control RKN has been demonstrated using earlier versions of commercial preparations; however, changes in fermentation and formulation technology required a reevaluation of the efficacy of the new biocontrol preparation (27). Results obtained with a novel glucose-based water-dispersible granule (WG) formulation of PL251 demonstrated efficacy for biological control of Meloidogyne incognita and M. hapla Chitwood on tomato, H. schachtii on sugar beet as well as the burrowing nematode Radopholus similis (Cobb) Thorne on banana (25).

Mode of action/interaction. Various mechanisms of action have been described for nematophaguous fungi, of which the eggparasitic fungi Pochonia chlamydosporia and Paecilomyces lilacinus and antagonists of motile stages of nematodes such as Arthrobotrys oligospora or Hirsutella rhossiliensis received the most attention (35). Mostly, the secretion of extracellular enzymes such as serine proteases or chitinases is required for successful parasitism of nematode eggs following an appressorium formation (35). The main mechanisms of action for PL251 have been described as direct infection of sedentary stages, in particular the egg stage, by direct penetration after formation of appressoria (14). The production of chitinases and proteases was identified as a key mode of action for PL251 against RKN but, in contrast to other isolates, strain 251 does not produce leucinotoxins which have been associated with biocontrol of $\operatorname{RKN}(20,21)$.

Interactions between antagonist and nematode. For some nematophaguous fungi, rhizosphere competence, host recognition, or host preference play an important role for their efficacy to control plant-parasitic nematodes. Fungal establishment in the rhizosphere, the proportion of egg-masses of Meloidogyne spp. exposed to the rhizoplane, and the growth rate relative to 
nematode development greatly affect the rhizosphere competence. For the rhizosphere-competent fungus V. chlamydosporium (syn. Pochonia chlamydosporia), root galls induced by Meloidogyne spp. as well as particular plant species stimulate growth and, consequently, enhance the biocontrol efficacy determined by the degree of egg parasitism (13). Furthermore, Mauchline et al. (31) demonstrated that different $P$. chlamydosporia isolates showed host preference on the infraspecific level. Conversely, all previous studies involving PL251 indicated that mechanisms of action other than rhizosphere competence are the key factors for biocontrol efficacy (25). It was demonstrated that PL251 was not able to proliferate in the rhizosphere of different host plants and is not believed to be an endophyte $(38,39)$. Furthermore, the level of egg mass colonization determined by standard isolation techniques was related to neither fungal density in soil nor the efficacy to reduce nematode damage on tomato roots. Using fluorescence, confocal laser scanning, and scanning electron microscopy, it could be demonstrated that, after inoculation of egg masses with conidia of PL251, growth of the fungus was restricted to the surface of the gall and no fungal growth was found on the gelatinous matrix covering the egg masses of $M$. incognita (26). This matrix is known to contain antimicrobial substances to protect the eggs inside the egg mass $(29,36)$.

Real-time quantitative polymerase chain reaction. Previous studies to quantify PL251 population densities in the rhizosphere of roots, on egg masses, or in soil samples have used direct isolation techniques on selective media. However, these methods are time consuming and may not have sufficient sensitivity or specificity to accurately quantify or locate fungal propagules (41). The abundance of fungi in the environment is difficult to asses and, therefore, molecular methods that rely on direct DNA extraction from plant samples avoiding the need for culture offer new opportunities to monitor individual species (5). Using realtime analysis and biomarker transformation to investigate the interaction of a nonpathogenic Fusarium oxysporum strain with $V$. dahliae revealed that competition for space and nutrients and, therefore, higher rhizosphere competence was the main mode of action (37). Similar results were obtained by Takaneka and coworkers (41), who investigated the interaction of Pythium oligandrum and bacterial wilt disease in the tomato rhizosphere by real-time polymerase chain reaction (PCR) and confocal laserscanning microscopy. For the detection and quantification of the nematophagous fungus Pochonia chlamydosporia, real-time PCR assays were developed based on Scorpion and TaqMan probes $(5,10)$. The latter not only could demonstrate that the fungus was able to uniformly colonize roots and galls from Meloidogyne spp. on tomato but also could differentiate the rhizosphere colonization ability of two $P$. chlamydosporia isolates. For Paecilomyces lilacinus, Atkins et al. (4) developed a real-time PCR assay for the quantification in field soil with a potential detection limit as low as $10 \mathrm{CFU} / \mathrm{g}$ of soil.

In order to fully evaluate the potential of a biological control agent, the importance of pathogen inoculum density and antagonist concentration for a dose-response relationship between the concentration of antagonist applied and the reduction of plant damage needs to be evaluated (34). This information may help in understanding factors affecting biocontrol interactions and can be useful in predicting biocontrol efficiency under varying conditions (30). Previous studies indicating that rhizosphere competence is not the key mode of action for PL251 were conducted under controlled conditions or with little or no variation in inoculum level (25). Field and commercial greenhouse studies had indicated that the concentration of PL251 in soil after application was lower than predicted but efficacy was still demonstrated; therefore, the goal of our studies was to investigate the effect of varying combinations of RKN inoculum density and antagonist dose on the biocontrol efficacy of PL251 applied as a single preplant soil treatment. Furthermore, a real-time PCR assay was used to determine colonization of RKN egg masses by PL251 and to investigate the role of rhizosphere competence for biocontrol efficacy.

\section{MATERIALS AND METHODS}

Nematode and plant material. Inoculum of $M$. incognita was prepared following the method of Hussey and Barker (15) by extracting nematode eggs from infected roots using a $1.5 \%$ $\mathrm{NaOCl}$ solution. The highly susceptible tomato cv. Furore (Solanum lycopersicum L.) was used in all experiments. Tomato seed were sown in 96-well multipot trays containing an unsterile field soil (silty clay, pH 5.2; $0.97 \%$ organic matter) and sand mixture $(1: 1, \mathrm{vol} / \mathrm{vol})$ plus $10 \%$ seedling substrate (Klaasmann, Germany). After 4 to 6 weeks, the seedlings were used for experiments.

Experimental design and conditions. For all experiments, a nonsterile field soil:sand mixture $(1: 1, \mathrm{vol} / \mathrm{vol})$, hereafter referred to as soil, was used. PL251 was applied using the commercial water-dispersible granule formulation with a concentration of $1 \times$ $10^{10}$ viable conidia/g of product $(27,28)$. The product was suspended in tap water and applied with a 30-ml suspension per $500 \mathrm{~cm}^{3}$ of soil and incorporated using a soil mixer (BeBa Mischtechnik, Germany). Afterwards, M. incognita inoculum containing $\approx 25 \%$ eggs with second-stage juveniles, $69 \%$ eggs in the embryonic stage, and $6 \%$ hatched second-stage juveniles was incorporated into the soil by hand.

Effects of M. incognita inoculum density and Paecilomyces lilacinus dose on biocontrol efficacy. A complete factorial design included all combinations of six inoculum levels of $M$. incognita $(0,100,200,400,800$, and 1,600 eggs and juveniles/ $100 \mathrm{~cm}^{3}$ of soil) and six doses of PL251 applied at $0,0.01,0.025$, $0.05,0.1$, and $0.2 \mathrm{~g}$ of water-dispersible granule formulation per $500 \mathrm{~cm}^{3}$ of soil. These doses corresponded to a concentration of $0,2 \times 10^{5}, 5 \times 10^{5}, 1 \times 10^{6}, 2 \times 10^{6}$, and $4 \times 10^{6} \mathrm{CFU}$ of PL251 per gram of soil. Soil treated with water only or water plus the $M$. incognita eggs and juvenile suspension served as controls. After treating and inoculating the bulk soil, equal amounts of $500 \mathrm{~cm}^{3}$ of soil were filled into $11-\mathrm{cm}$-diameter plastic pots and moistened to field capacity. To confirm the correct concentration of PL251 in soil, samples from individual pot replicates of each treatment were taken and pooled, and the number of $\mathrm{CFU} / \mathrm{g}$ of soil was determined by dilution plating $(27,38)$. Pots were then transferred to a greenhouse bench, covered with plastic wrap to prevent rapid drying of the treated soil, and incubated with greenhouse temperatures set at $25 \pm 1{ }^{\circ} \mathrm{C}$ without additional light. The pots were left on the greenhouse bench for 6 days to allow the fungus to establish in the soil and to infect the nematode inoculum. During this time, a sufficient moisture level was maintained to ensure viability of the nematode inoculum. On day seven, one tomato plant was then transplanted into each pot. To guarantee direct exposure to treated soil, the roots of the tomato seedlings were gently washed free of soil before transplanting. Starting 1 week after transplanting, tomato plants were fertilized biweekly with a 20-10-15 (N-P-K) fertilizer solution. The experiment was performed with five replicates per treatment and conducted twice.

Data collection. Plants were harvested 10 weeks after transplanting, and shoot fresh weight was determined. After careful uprooting and washing the roots free of soil, root fresh weight was measured and nematode damage was determined by rating root galling on the 0 -to- 10 scale $(0=$ no galls and $10=$ dead plant) of Bridge and Page (8). In addition, the number of egg masses per root system was determined after staining with a Phloxine B solution ( $0.015 \%$; Sigma). After counting the number of egg masses per root, 10 egg masses were randomly collected from each replicate, pooled, transferred to a 1.5-ml Eppendorf tube, and stored at $-20^{\circ} \mathrm{C}$ until further use. In this way, $100 \mathrm{M}$. incognita egg masses were collected per treatment, resulting in a 
total of 3000 egg masses used in real-time PCR assays for both experiments.

Quantification of PL251 DNA on $M$. incognita egg masses. To investigate the interaction of PL251 with the RKN $M$. incognita, the quantification of the fungus on the egg masses was done using a modified real-time PCR protocol developed by Atkins et al. (4). Real-time PCR quantification was performed using TaqManPCR primers and probe PLrtF 5' GAC CCA AAA CTC TTT TTG CAT TAC G 3', PLrtR 5' AGA TCC GTT GTT GAA AGT TTT GAT TCA TT GTT TTG 3', and PLrtP 5' FAM CCG GCG GAA TTT CTT CTC TGA GTT GC TAMRA 3', which were designed from the internal transcribed spacer (ITS) region of the fungal genome. The fluorogenic probe (PLrtP) was labelled at the $5^{\prime}$ end with the fluorogenic reporter dye 6-carboxyfluorescein (FAM), and the $3^{\prime}$ end was modified with the quencher dye 6-carboxy-tetramethylrhodamine (TAMRA). Reactions were performed using the TaqMan universal PCR master mix (Applied Biosystems). To generate a standard curve for realtime PCR quantification, 10 egg masses each were taken from roots of a tomato plant (cv. Furore) infected with $M$. incognita. After adding $1 \times 10^{7}$ conidia of PL251, total DNA was extracted from egg masses in triplicate with an UltraClean plant DNA isolation kit (MO-BIO Laboratories, Inc.). Afterwards, DNA was serially diluted in sterile MilliQ water so that $3 \mu$ represented $1 \times$ $10^{5}$ to $1 \times 10^{4}$ to a final dilution of $10 \mathrm{CFU}$ per egg mass. DNA prepared from the $M$. incognita egg masses obtained from the untreated, inoculated plants was used as a negative control.

To evaluate the extraction efficacy of PL251 DNA in the presence of the egg masses, a second standard curve was prepared by extracting DNA from $1 \times 10^{7}$ conidia of PL251 only. After DNA was extracted from conidia in triplicate, serial dilutions were prepared from each sample of template DNA in sterile MilliQ water as described above so that $3 \mu$ represented $1 \times 10^{5}$ to $1 \times 10^{4}$ to a final dilution of $10 \mathrm{CFU}$ per reaction.

Real-time PCR was performed in fast optical 96-well 0.1-ml reaction plates covered with optical adhesive cover (Applied Biosystems) with the automated ABI Prism 7500 sequence detector (Applied Biosystems). After optimizing the primer and probe concentrations according to the supplier's recommendations, primers PLrtF and PLrtR were included at a final concentration of $900 \mathrm{nM}$ each and the TaqMan probe (PLrtP) was used at $225 \mathrm{nM}$. Total reaction volume was $12 \mu \mathrm{l}$. The thermal cycle protocol for PCR amplification was used according to Atkins et al. (4) and reactions were performed in triplicate. A standard curve was generated by using data derived from the serial dilution of PL251 from egg masses. For each real-time assay, a serial dilution to generate a standard curve was included and the cycle threshold $\left(\mathrm{C}_{\mathrm{T}}\right)$ was automatically calculated and analyzed by the Applied Biosystems 7500 software (version 2.0.2).

Specificity of the real-time PCR assay. To evaluate the specificity of the primers and probe used in this study, real-time PCR assays were conducted with DNA from 12 different isolates of antagonistic fungi belonging to eight species (Table 1). A $P$. lilacinus isolate (CBS 431.87) obtained from the Centraalbureau voor Schimmelcultures culture collection (Utrecht, The Netherlands) was included in this study as a positive control. Genomic DNA was extracted from the fungal isolates using the UltraClean plant DNA isolation kit as described above. A serial dilution of PL251 template DNA from $5 \mathrm{ng}$ to $5 \mathrm{fg}$ was used to generate a standard curve. Genomic DNA of the fungal isolates was adjusted using sterile MilliQ water to a final concentration of $0.45 \mathrm{ng} /$ reaction. Reactions were performed in triplicate and the experiment was conducted twice.

Data analysis. Data were analyzed using SPSS for Windows (version 15.0; SPSS Inc., IL). After confirming homogeneity of variances, data from the two experiments were pooled before further analysis. A two-way analysis of variance was used to assess the effects of $M$. incognita inoculum level, PL251 dose, and their interactions on the parameters shoot and root fresh weight. Furthermore, the parameters root gall index, the number of egg masses per root, and the biocontrol efficacy (percent reduction of nematode damage compared with the untreated, inoculated control calculated according to Abbott) (1) were analyzed. To detect dose-response relationships between the parameters root galling and egg masses and the concentration of the biocontrol product applied, a logistic dose-response curve model was used at each tested inoculum level as described previously (27).

To detect significant relationships between parameters gall index, egg masses per root, percent biocontrol efficacy, and density of PL251, Pearson product moment correlation coefficients were calculated (32). For parameters where strong and significant relationships were found, curve fitting by nonlinear regression was performed using Sigma plot for Windows (version 9.01; Systat Software Inc.).

\section{RESULTS}

Concentration of PL251 in soil. For both experiments, CFU numbers per gram of soil determined at the set-up of the experiment by serial dilution plating corresponded to the expected concentration (data not shown). Therefore, gram of product per $500 \mathrm{~cm}^{3}$ soil is used for the dose of PL251 throughout the text.

Effects on shoot and root weight. Two-factor analysis for the parameters shoot and root fresh weight revealed significant effects of the inoculum level and dose applied on the shoot fresh weight

TABLE 1. Specificity of primers PLrt F and PLrtR and probe PLrtP in a real-time polymerase chain reaction assay using template DNA

\begin{tabular}{|c|c|c|c|c|}
\hline Species name & Strain & Source $^{w}$ & $\mathrm{C}_{\mathrm{T}} \pm \mathrm{SD}^{\mathrm{x}}$ & Template $(\mathrm{fg})^{\mathrm{y}}$ \\
\hline Paecilomyces lilacinus & 431.87 & CBS & $23.92 \pm 1.41$ & $168,600 \pm 18,600$ \\
\hline Clonostachys rosae & IK761 & $\mathrm{KVL}^{\mathrm{z}}$ & NS & $\ldots$ \\
\hline C. rosae & $224.72 \mathrm{~A}$ & CBS & NS & $\ldots$ \\
\hline Lecanicillium lecanii & 101283 & CBS & $40.90 \pm 3.67$ & $3.99 \pm 2.74$ \\
\hline Pochonia chlamydosporia & 102066 & CBS & NS & $\ldots$ \\
\hline Paecilomyces farinosus & KVL00-124 & KVL & $39.16 \pm 1.25$ & $8.22 \pm 4.16$ \\
\hline P. farinosus & KVL04-021 & KVL & $38.97 \pm 1.95$ & $13.60 \pm 10.1$ \\
\hline$P$. fumosoroseus & 864.96 & CBS & NS & $\ldots$ \\
\hline Trichoderma harzianum & 245.93 & CBS & $41.20 \pm 2.69$ & $4.82 \pm 4.23$ \\
\hline T. virens & 498.84 & CBS & NS & $\ldots$ \\
\hline
\end{tabular}

${ }^{\mathrm{w}}$ CBS = Centraalbureau voor Schimmelcultures, Utrecht, The Netherlands and KVL = University of Copenhagen, Faculty of Life Sciences, Department of

Ecology, Section of Zoology, Thorvaldsensvej 40, DK-1871 Frederiksberg.

${ }^{x}$ Cycle threshold $\left(\mathrm{C}_{\mathrm{T}}\right)$ values \pm standard deviation $(\mathrm{SD}) ; \mathrm{NS}=$ no fluorescence signal detected or $\mathrm{C}_{\mathrm{T}}$ values $>42$.

${ }^{\mathrm{y}}$ According to standard curve using $5 \mathrm{ng}$ to $5 \mathrm{f}$ of template DNA from PL251 $\left(y=-3.421 x+31.528 ; r^{2}=0.955\right)$.

z Jensen et al. (17). 
(Table 2). However, none of the tested inoculum levels resulted in a shoot weight significantly different from the untreated, noninoculated control (data not shown). For the factor dose, the shoot weight was significantly greater compared with the untreated control after treatment with $0.01,0.05$, and $0.2 \mathrm{~g}$ of PL251. The parameter root fresh weight was affected by the dose applied $(P<$ 0.001 ) but not by the inoculum level (Table 2). For the parameters shoot and root weight, no significant interaction between the main factors inoculum level and dose was found (Table 2). For the parameters gall index and number of egg masses per root, the inoculum level as well as the dose of PL251 applied to soil had a highly significant effect. In addition, significant interactions between the main factors were observed (Table 2).

Dose-response relationships. When data from the experiments were analyzed using the logistic dose-response model, no relationship between the dose of product applied and the development of the nematode damage parameters gall index and number of egg masses per root could be established (data not shown). The effect of different doses of PL251 applied at five inoculum levels on the parameters gall index and number of egg masses per root is shown in Figure 1A and B. For all five doses of PL251 tested, the lowest gall index ratings and egg mass numbers were found at 100,200 , and 400 eggs and second-stage juveniles $\left(E+J_{2}\right) / 100$ $\mathrm{cm}^{3}$. At 800 and $1,600 \mathrm{E}+\mathrm{J}_{2} / 100 \mathrm{~cm}^{3}$, no further increase in gall index development and number of egg masses per root was found in the untreated, inoculated controls compared with an inoculum level of $400 \mathrm{E}+\mathrm{J}_{2}$ or less. Conversely, in soil treated with 0.01 to $0.1 \mathrm{~g}$ of PL251, the gall index rating and number of egg masses per root were increased at 800 and $1,600 \mathrm{E}+\mathrm{J}_{2} / 100 \mathrm{~cm}^{3}$, respectively (Fig. 1A and B). At $1,600 \mathrm{E}+\mathrm{J}_{2} / 100 \mathrm{~cm}^{3}$, treatment with $0.2 \mathrm{~g}$ of product resulted in a gall index and a number of egg masses per root not different from the untreated, inoculated control.

When averaged over the main factors, the level of inoculum had a highly significant effect on the development of the gall index and the number of egg masses per root (Table 3). The corresponding biocontrol efficacy of the PL251 soil treatment was also affected by the inoculum level. At 100 to $400 \mathrm{E}+\mathrm{J}_{2} / 100 \mathrm{~cm}^{3}$, efficacy was 42 to $51 \%$ whereas, at 800 and $1,600 \mathrm{E}+\mathrm{J}_{2} / 100 \mathrm{~cm}^{3}$, the efficacy was lower, at $22 \%(P<0.0001)$ (Table 3$)$. A similar effect was observed for the efficacy in reducing the number of egg masses per root. At 100 to $400 \mathrm{E}+\mathrm{J}_{2} / 100 \mathrm{~cm}^{3}$, biocontrol efficacy was 86 to $82 \%$ whereas, at 800 and $1,600 \mathrm{E}+\mathrm{J}_{2} / 100 \mathrm{~cm}^{3}$, the efficacy was lower, at 42 and $30 \%$, respectively $(P<0.0001)$ (Table 3). When averaged over the PL251 soil treatment, each dose reduced the gall index when compared with the untreated, inoculated control $(P<0.0001)$. Treatment with $0.2 \mathrm{~g}$ of PL251 resulted in an intermediate gall index significant different from both the untreated control and soil treatments with $\leq 0.1 \mathrm{~g}$ of PL251. In addition, the biocontrol efficacy at $0.2 \mathrm{~g}$ of PL251 was wirh $25 \%$ lower compared with soil treated with $\leq 0.1 \mathrm{~g}$ (36 to $45 \%, P=0.003$ ) (Table 3). Similar results were obtained for the

TABLE 2. $P$ values of the two-way analysis of variance of the main factors Meloidogyne incognita inoculum level, Paecilomyces lilacinus strain 251 dose, and their interactions on the response variables tomato (cv. Furore) shoot and root fresh weight, gall index, and egg masses per root

\begin{tabular}{lcccc}
\hline & \multicolumn{4}{c}{ Response variable } \\
\cline { 2 - 5 } Factor & $\begin{array}{c}\text { Shoot fresh } \\
\text { weight }\end{array}$ & $\begin{array}{c}\text { Root fresh } \\
\text { weight }\end{array}$ & $\begin{array}{c}\text { Gall } \\
\text { index }^{\mathrm{x}}\end{array}$ & $\begin{array}{c}\text { Egg masses } \\
\text { per root }\end{array}$ \\
\hline Inoculum level $^{\mathrm{y}}$ & 0.045 & 0.263 & $<0.001$ & $<0.001$ \\
Dose $^{\mathrm{z}}$ & 0.012 & $<0.001$ & $<0.001$ & $<0.001$ \\
Inoculum $\times$ dose $^{0}$ & 0.991 & 0.864 & 0.026 & 0.002 \\
\hline
\end{tabular}

${ }^{x}$ Gall index according to Bride and Page (8).

${ }^{\mathrm{y}}$ Level: $0,100,200,400,800$ and 1,600 eggs and second stage juveniles/ $100 \mathrm{~cm}^{3}$.

${ }^{\mathrm{z}}$ Dose: $0,0.01,0.025,0.05,0.1$, and $0.2 \mathrm{~g}$ of product per $500 \mathrm{~cm}^{3}$ of soil. number of egg masses per root, where the biocontrol efficacy at a dose of $\leq 0.1 \mathrm{~g}$ was 63 to $71 \%$. A lower biocontrol efficacy was determined (48\%) when a dose of $0.2 \mathrm{~g}$ of PL251 per $500 \mathrm{~cm}^{3}$ of soil was applied $(P=0.003)$ (Table 3$)$.

Quantification of PL251 by real-time PCR. For both standard curves, a strong linear inverse relationship between the $\mathrm{C}_{\mathrm{T}}$ values and the $\log _{10} \mathrm{CFU}$ concentration (with and without $M$. incognita egg masses) over five orders of magnitude were found (Fig. 2). The two standard curves were different in their intercept but comparison of the slopes according to Zar (43) revealed no significant difference $(P<0.05)$. This indicated that the efficacy of DNA extraction from PL251 conidia in the presence of $M$. incognita egg masses was slightly reduced; however, no PCR inhibitors were present that interfered with the quantification. To avoid an underestimation of the PL251 density, serially diluted DNA extracted from $M$. incognita egg masses together with PL251 conidia was included in each real-time PCR assay to generate a standard curve to automatically calculate the concentration of PL251 in log CFU per egg mass. With this method, it was possible to detect as low as $10 \mathrm{CFU}$ of PL251 per $M$. incognita egg mass (Fig. 2).

Density of PL251 on $M$. incognita egg masses. The density of PL251 on $M$. incognita egg masses from plants treated with different doses at five different inoculum levels was determined by real-time PCR assays. Over all treatment and inoculum levels, 60 of 250 samples yielded no fluorescence signals or $C_{T}$ values above the detection limit for PL251 (data not shown). For the
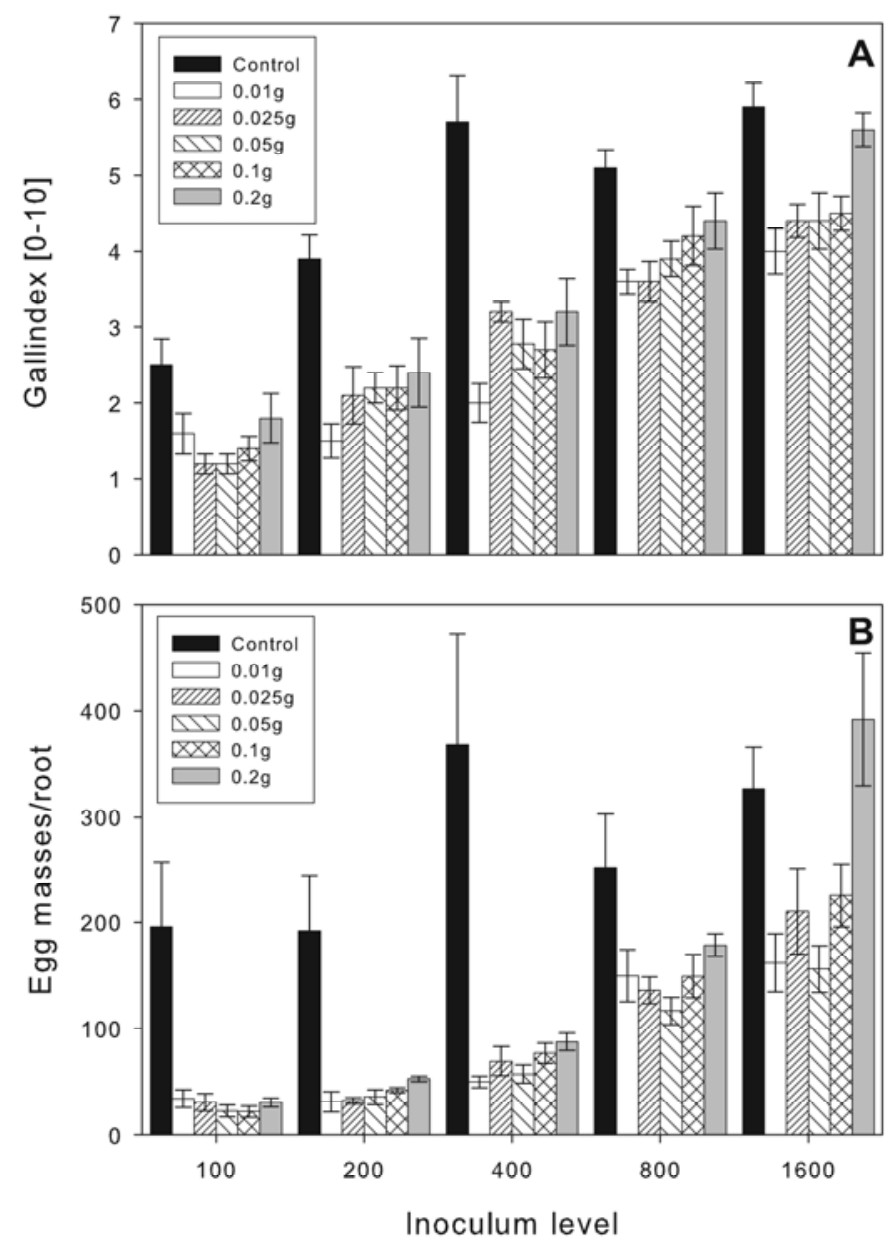

Fig. 1. Effects of the Meloidogyne incognita inoculum level (in eggs and second stage juveniles per $100 \mathrm{~cm}^{3}$ of soil) and the dose of Paecilomyces lilacinus strain 251 applied to soil (in grams per $500 \mathrm{~cm}^{3}$ ) on gall index and number of egg masses on tomato cv. Furore after 10 weeks in a greenhouse. Bars represent means \pm standard error from two independent experiments. 
remaining samples, the fluorescence signals were intensive enough to calculate densities of PL251 ranging from $\log 1.0$ to $\log 3.2$ CFU per $M$. incognita egg mass based on the standard curve (Fig. 2), which corresponded to $\approx 10$ to $1.62 \times 10^{3} \mathrm{CFU}$. In addition, for 17 of 50 samples $(24 \%)$ from egg masses obtained from the untreated, inoculated control plants, weak fluorescence signals were detected. However, these $\mathrm{C}_{\mathrm{T}}$ values corresponded on average to a density of less than $5 \mathrm{CFU}$ per egg mass (data not shown). Pearson product moment correlations revealed only one strong relationship between the density of PL251 on M. incognita egg masses and the rate of product applied $(r=0.646, P<$ $0.0001)$. Therefore, data were further analyzed using nonlinear regression. An exponential model effectively described the relationship between the dose of product applied in grams per $500 \mathrm{~cm}^{3}$ of soil and the density of PL251 per egg mass in log CFU quantified by real-time PCR $\left(r^{2}=0.854 ; P=0.0084\right)$ (Fig. 3 ). The density of PL251 on $M$. incognita egg masses rapidly increased with an increase in dose from 0.01 to $0.05 \mathrm{~g}$ per $500 \mathrm{~cm}^{3}$ but no further increase was found after application of 0.1 and $0.2 \mathrm{~g}$ of product, respectively.

Specificity of real-time PCR analysis. The DNA from 12 different strains of antagonistic fungi belonging to eight species (Table 1) was tested for cross reactions of the primers and probe used in real-time PCR assays. For the positive control (P. lilacinus strain CBS431.87), a strong fluorescence signal was detected, whereas no or only weak signals were obtained for 10 of 12 strains tested (Table 1). For the two P. farinosus strains KVL00124 and KVL04-021, $\mathrm{C}_{\mathrm{T}}$ values of 39.2 and 38.9, respectively, were obtained which, based on the standard curve, corresponded to 8.2 and 13.6 fg of PL251 genomic DNA, respectively. However, these $C_{T}$ values corresponded to fluorescence signals representing the detection limit of 10 CFU of PL251 per $M$. incognita egg mass (Fig. 2).

\section{DISCUSSION}

Effects of $M$. incognita inoculum density and $P$. lilacinus dose on biocontrol efficacy. In contrast to the previous doseresponse studies conducted under more controlled environmental conditions and at a fixed inoculum density (27), significant doseresponse relationships could be established for none of the doseinoculum combinations tested. This difference might be, in part, explained by the higher temperatures at which these experiments were conducted. Although the greenhouse temperature was set at $25 \pm 1{ }^{\circ} \mathrm{C}$, maximum daily temperatures reached 28 to $33^{\circ} \mathrm{C}$, resulting in an average daily temperature of $28 \pm 1.5^{\circ} \mathrm{C}$ during the experiment, which is the optimum range for germination and growth of PL251 (22,28). Furthermore, high temperatures are also favorable for the development and damage potential of the tropical RKN $M$. incognita (11), which also plays a role in the interactions between biocontrol agent and nematode. When models are used to describe dose-response relationships in pathogen biocontrol, they are mostly based on biocontrol titration at a single pathogen dose (34). The problem with this approach can be that, at a low inoculum level over control and at too high pathogen densities, a lack of control can occur. Previous studies were conducted at a fixed inoculum density of 1,000 M. incognita $\mathrm{E}+\mathrm{J}_{2} / 100 \mathrm{~cm}^{3}$ of soil, which was obviously the optimum level for testing doses of 0.01 to $0.2 \mathrm{~g} / 500 \mathrm{~cm}^{3}$ of soil. However, in these studies, a dose of $0.2 \mathrm{~g}$ of the glucose-based formulation of PL251 resulted in a lower biocontrol efficacy when compared with PL251 conidia applied without formulation (27). The reduction in efficacy might be due to the higher glucose concentration in soil which inhibits serine protease activity of PL251 $(6,20)$. Serine protease activity is critical in reducing the initial soil inoculum because it inhibits egg development and, consequently, hatching of infective juveniles (6).

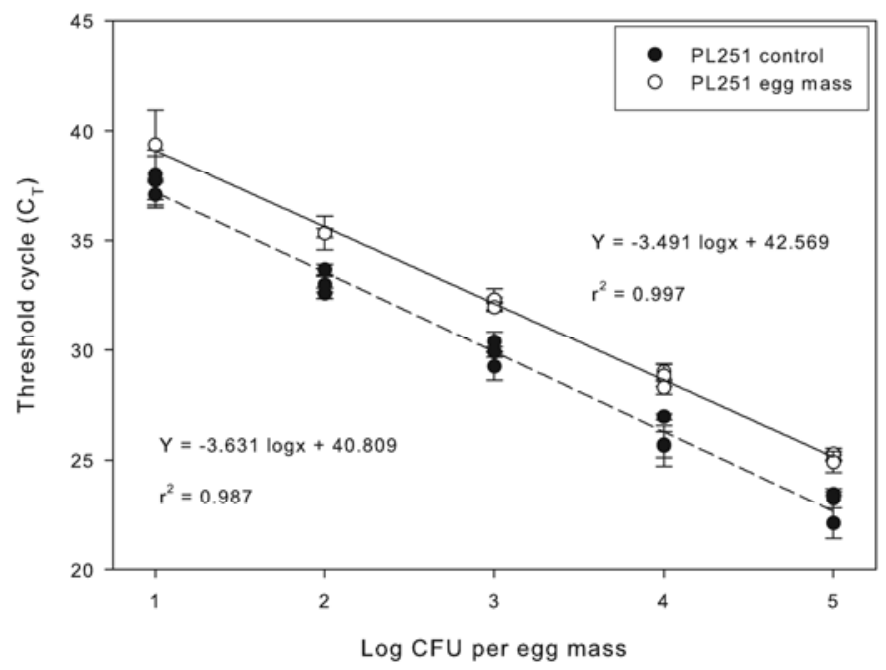

Fig. 2. Standard curves of Paecilomyces lilacinus strain 251 DNA extracted with (○) and without Meloidogyne incognita egg masses (O) in real-time polymerase chain reaction assays. Threshold cycle values are plotted against DNA per reaction representing $\log 5$ to $\log 1 \mathrm{CFU}$ per egg mass. Data points are means ( \pm standard error) from three independent experiments.

TABLE 3. Effects of the main factors Meloidogyne incognita inoculum level and the dose of Paecilomyces lilacinus strain 251 (PL251) applied to soil on gall index, number of egg masses on tomato cv. Furore, and biocontrol efficacy after 10 weeks in a greenhouse $\mathrm{v}^{\mathrm{v}}$

\begin{tabular}{|c|c|c|c|c|}
\hline Inoculum, dose & Gall index ${ }^{\mathrm{w}}$ & Biocontrol efficacy $(\%)^{\mathrm{x}}$ & Egg masses per root & Biocontrol efficacy $(\%)^{\mathrm{x}}$ \\
\hline \multicolumn{5}{|l|}{ Inoculum levely } \\
\hline Control & $\ldots$ & $\ldots$ & & \\
\hline 100 & $1.6 \pm 0.11 \mathrm{e}$ & $42.4 \pm 4.0 \mathrm{a}$ & $56.0 \pm 12.9 \mathrm{~d}$ & $85.7 \pm 1.5 \mathrm{a}$ \\
\hline 200 & $2.4 \pm 0.16 \mathrm{~d}$ & $46.7 \pm 3.7 \mathrm{a}$ & $64.4 \pm 11.4 \mathrm{~d}$ & $79.4 \pm 1.4 \mathrm{a}$ \\
\hline 400 & $3.3 \pm 0.22 \mathrm{c}$ & $51.3 \pm 2.7 \mathrm{a}$ & $119.3 \pm 22.8 \mathrm{c}$ & $81.6 \pm 1.2 \mathrm{a}$ \\
\hline 800 & $4.1 \pm 0.13 b$ & $22.7 \pm 2.6 b$ & $164.0 \pm 11.7 \mathrm{~b}$ & $42.1 \pm 3.1 b$ \\
\hline 1,600 & $4.8 \pm 0.14 \mathrm{a}$ & $22.4 \pm 2.4 \mathrm{~b}$ & $245.4 \pm 19.0 \mathrm{a}$ & $29.8 \pm 6.4 \mathrm{c}$ \\
\hline \multicolumn{5}{|l|}{ PL251 dose $(\mathrm{g})^{\mathrm{z}}$} \\
\hline Control & $4.6 \pm 0.25 \mathrm{~A}$ & $\ldots$ & $266.9 \pm 29.8 \mathrm{~A}$ & $\ldots$ \\
\hline 0.01 & $2.5 \pm 0.18 \mathrm{C}$ & $44.8 \pm 3.5 \mathrm{~A}$ & $84.1 \pm 11.1 \mathrm{C}$ & $69.3 \pm 3.8 \mathrm{~A}$ \\
\hline 0.025 & $2.9 \pm 0.19 \mathrm{C}$ & $39.7 \pm 3.0 \mathrm{~A}$ & $95.7 \pm 13.1 \mathrm{C}$ & $66.0 \pm 4.1 \mathrm{~A}$ \\
\hline 0.05 & $2.9 \pm 0.20 \mathrm{C}$ & $38.9 \pm 2.9 \mathrm{~A}$ & $78.1 \pm 9.2 \mathrm{C}$ & $71.7 \pm 3.0 \mathrm{~A}$ \\
\hline 0.10 & $3.0 \pm 0.21 \mathrm{C}$ & $36.3 \pm 3.4 \mathrm{~A}$ & $103.2 \pm 12.9 \mathrm{C}$ & $63.5 \pm 4.1 \mathrm{~A}$ \\
\hline 0.20 & $3.5 \pm 0.25 \mathrm{~B}$ & $25.8 \pm 4.5 \mathrm{~B}$ & $148.4 \pm 22.5 \mathrm{~B}$ & $48.4 \pm 6.8 \mathrm{~B}$ \\
\hline
\end{tabular}

${ }^{v}$ Within a main factor and column, treatments followed by the same letter are not significantly different according to Student-Newman-Keuls test at $P \leq 0.05$.

${ }^{\mathrm{w}}$ Gall index according to Bride and Page (8).

${ }^{x}$ Percent reduction of gall index and number of egg masses per root calculated according to Abbott (1).

${ }^{y}$ Eggs and second stage juveniles $/ 100 \mathrm{~cm}^{3}$.

${ }^{\mathrm{z}}$ Dose in grams of product per $500 \mathrm{~cm}^{3}$ of soil. 
For the nematophagous fungus Pochonia chlamydosporia, Kerry (18) stated that multiple interactions affected the rhizosphere competence and, consequently, the biocontrol efficacy toward RKN. The host plant and presence of the nematode target which trigger growth in the rhizosphere allowed for a sufficient biocontrol efficacy at low to medium inoculum densities. However, at high inoculum densities, most of the RKN population developed inside the roots and, therefore, was not affected by this rhizosphere-competent fungus. In contrast, PL251 was never found to proliferate in the rhizosphere of different host plants nor was endophytic colonization of roots or galls found but it did show a sufficient biocontrol efficacy at high inoculum densities (25). Two-factorial analysis revealed a significant interaction between the dose of PL251 applied to soil and the inoculum level for the parameters gall index and egg masses per root. This interaction might be due to the fact that the PL251 application reduced the higher nematode inoculum to a level where conditions for root penetration, root galling, and production of egg masses was again at the optimum for $M$. incognita. A similar effect was described by Bourne and Kerry (7), who found that the efficacy of $V$. chlamydosporium (syn. P. chlamydosporia) to reduce nematode damage and reproduction was lower when a higher antagonist dose was applied at an elevated level of $M$. incognita inoculum. In contrast to previous dose-response studies (27), it was demonstrated that sufficient biocontrol efficacy was achieved with a single PL251 preplanting soil application at a dose of $0.01 \mathrm{~g} / 500 \mathrm{~cm}^{3}\left(2 \times 10^{5} \mathrm{CFU} / \mathrm{g}\right.$ of soil $)$. This confirms studies under field and commercial greenhouse conditions where a preplanting application of PL251 followed by post-planting drenches throughout the growing season provided significant control of RKN $(2,23)$. Our studies show a strong reduction in the galling index and the number of egg masses per after PL251 soil treatment at inoculum densities up to $400 \mathrm{E}+\mathrm{J}_{2} / 100 \mathrm{~cm}^{3}$. Under field conditions, at inoculum densities of 100 to $400 \mathrm{E}+\mathrm{J}_{2} / 100$ $\mathrm{cm}^{3}$ of soil, severe yield losses will occur for all main host plants of $M$. incognita (12).

Specificity of real-time PCR analysis. In this study, we examined a real-time PCR assay for the quantification of PL251 on $M$. incognita egg masses from tomato. Although weak fluorescence signals were detected with genomic DNA from Gliocladium catenulatum, Paecilomyces farinosus, P. fumoso-

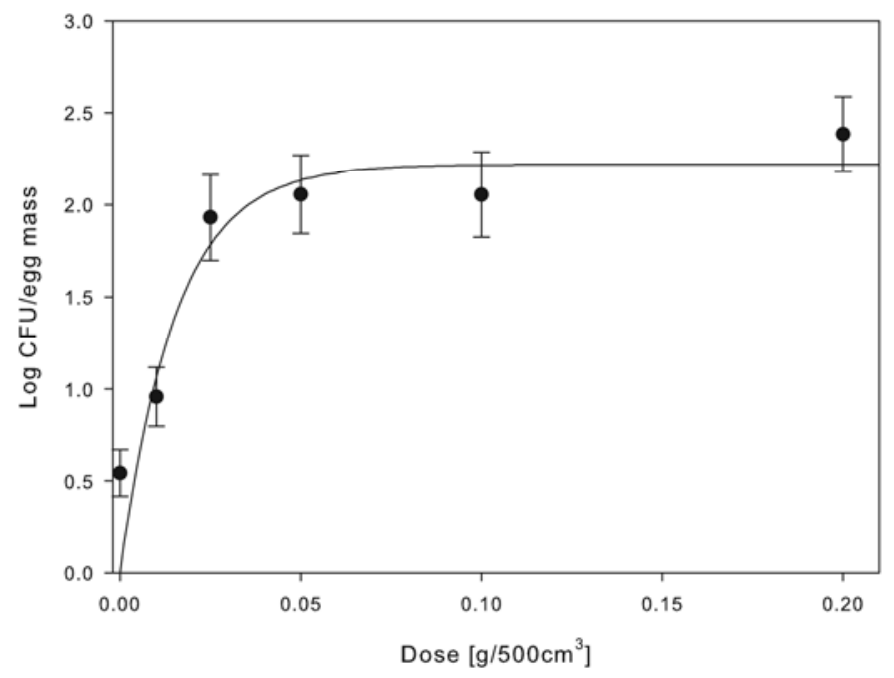

Fig. 3. Relationship between the dose of product applied to soil in grams per $500 \mathrm{~cm}^{3}$ and the density of Paecilomyces lilacinus strain 251 (PL251) per Meloidogyne incognita egg mass in $\log \mathrm{CFU}$ as determined by real-time polymerase chain reaction assays. Data points are means of 30 replicates ( \pm standard error); $y=2.2178[1-\exp (-62.2667 x)]$, where $y$ is the density of PL251 per egg mass in $\log$ CFU and $x$ is the rate of product applied in grams per $500 \mathrm{~cm}^{3}$ of soil; $r^{2}=0.854(P=0.0084)$. roseus, and Trichoderma harzianum (Table 1), the $\mathrm{C}_{\mathrm{T}}$ values corresponded to the detection limit of PL251 on M. incognita egg masses (Fig. 2). Similar effects were found by Takaneka et al. (41), who detected low but significant fluorescence signals in untreated, unsterile field soil using a real-time assay for the quantification of Pythium oligandrum in the rhizosphere of tomato. However, they concluded that the specificity of their assay was still sufficient because a significant increase in the fluorescence signal was detected after inoculation with P. oligandrum. The real-time PCR TaqMan assay we used and modified was first developed by Atkins et al. (4) for detection and quantification of Paecilomyces lilacinus in soil from two different field sites. However, they did not test specificity with other fungal antagonists that could be present in field soils and colonize RKN egg masses. To solve this problem of unspecific reactions of the primer and probe set, research is currently being undertaken to develop a highly specific primer and probe set based on TaqMan minor grove binding probe design which can significantly enhance the specificity of real-time PCR assays (42).

Quantification of PL251 by real-time PCR. The comparison of the standard curves with genomic DNA obtained from PL251 conidia in the presence or absence of $M$. incognita egg masses revealed a slight reduction in the $\mathrm{C}_{\mathrm{T}}$ values measured but the slopes of the two standard curves were not different (Fig. 2). Background DNA and other co-extracted contaminants can affect the sensitivity of real-time PCR assays (9). In particular, humic acids are known to inhibit Taq polymerase in PCR and to interfere with detection assays. The reduced $\mathrm{C}_{\mathrm{T}}$ values in the standard curve obtained from PL251 conidia in the presence of $M$. incognita egg masses were caused by a reduced DNA extraction efficacy. Modification of the DNA extraction protocol might lead to higher DNA yield but can also allow for more release of background DNA and inhibitors (9). However, in our studies, a detection limit of $10 \mathrm{CFU}$ per egg mass was obtained, which represents sufficient sensitivity. Similarly, real-time PCR assays developed for detection and quantification of other nematodeantagonistic fungi such as Pochonia chlamydosporia were highly sensitive, with detection limits as low as 5 CFU reaction (5).

Density of PL251 on M. incognita egg masses. In our studies, egg mass colonization by PL251 as determined by real-time PCR assays was only correlated to the dose of product applied. These findings confirm investigations using culture-based methods which also revealed that there was no relationship between biocontrol efficacy, the concentration of the fungus in the soil, and the incidence of PL251 on RKN egg masses $(23,24)$. A further approach to understand the interaction of PL251 with RKN using confocal laser-scanning microscopy, fluorescence, and scanning electron microscopy revealed no colonization of the gelatinous matrix protecting $M$. incognita egg masses (26). The role of the gelatinous matrix in the suppression of nematode-antagonistic microorganisms was described by Orion et al. (36), who reported the antimicrobial properties of this substance. Furthermore, Kok et al. (29) found that the microbial community colonizing the gelatinous matrix of RKN harbored a great number of bacterial isolates expressing direct antagonism against the nematophagous fungus $V$. chlamydosporium. Conversely, Sharon et al. (40) demonstrated the importance of the gelatinous matrix in the parasitism of $M$. javanica by Trichoderma spp. Growth and parasitism of eggs and juveniles by $T$. asperellum was positively affected by the presence of the gelatinous matrix but parasitism by $T$. harzianum was inhibited. Using real-time quantitative PCR, significant differences in the rhizosphere competence of $P$. chlamydosporia var. catenulata and var. chlamydosporia isolates were described (5). In contrast to our findings, the amount of initial fungal inoculum applied to soil did not affect the amount of the DNA recovered from $M$. incognita egg masses, although differences in nematode gall and egg mass colonization were detected (5). 
In conclusion, our data demonstrate, although dose-response relationships could not be established as expected, that the application of PL251 to the soil 6 days before transplanting tomato resulted in a high level of $M$. incognita control at initial inoculum levels that, under field conditions, would cause significant damage and yield loss. In contrast to previous studies, we found a concentration of $2 \times 10^{5} \mathrm{CFU} / \mathrm{g}$ soil sufficient for biological control of $M$. incognita on tomato. Quantification of PL251 by real-time PCR proved to be a very useful technique to understand the relationships between nematode inoculum density, dose of fungus applied, and biocontrol efficacy against the RKN $M$. incognita. It was demonstrated that, in contrast to other nematode-antagonistic fungi, rhizosphere competence of PL251 is not the key factor for successful biocontrol. This feature can be an advantage for commercial uses because the nonspecialized mode of action allows a wider range of applications compared with other antagonists where cropping sequences with host and nonhost plant species are needed for successful biocontrol (18). The reduction of the nematode inoculum in soil by PL251 prior to transplanting is critical to prevent initial nematode damage on plants. Studies on the effect of plant species and the presence of the nematode host on the persistence of PL251 in soil revealed no effect on the abundance or survival; therefore, repeated applications are necessary to ensure reduction of RKN damage throughout a cropping season $(23,38,39)$. This biocontrol fungus represents an interesting tool for an integrated management program for plant-parasitic nematodes. However, application of PL251 using adapted formulations which would increase colonization of egg masses and, in particular, the gelatinous matrix could lead to further enhancement of biocontrol efficacy and warrants further research.

\section{ACKNOWLEDGMENTS}

We thank J. Samietz for statistical advice and Prophyta Biologischer Pflanzenschutz $\mathrm{GmbH}$, Germany, for providing product samples of MeloCon WG.

\section{LITERATURE CITED}

1. Abbott, W. S. 1925. A method of computing the effectiveness of an insecticide. J. Econ. Entomol. 18:265-267.

2. Anastasiadis, I. A., Giannakou, I. O., Prophetou-Athanasiadou, D. A., and Gowen, S. R. 2008. The combined effect of a biocontrol agent Paecilomyces lilacinus, with various practices for the control of root-knot nematodes. Crop Prot. 27:352-361.

3. Anonymous. 2008. Commission Directive 2008/44/EC of 4 April 2008 amending Council Directive 91/414/EEC to include benthiavalicarb, boscalid, carvone, fluoxastrobin, Paecilomyces lilacinus and prothioconazole as active substances. Off. J. Eur. Union L94:13-15.

4. Atkins, S. D., Clark, I. M., Pande, S., Hirsch, P. R., and Kerry, B. K. 2005. The use of real-time PCR and species-specific primers for the identification and monitoring of Paecilomyces lilacinus. FEMS Microbiol. Ecol. 51:257-264.

5. Atkins, S. D., Peteira, B., Clark, I. M., Kerry, B. R., and Hirsch, P. R. 2009. Use of real-time quantitative PCR to investigate root and gall colonisation by co-inoculated isolates of the nematophaguous fungus Pochonia chlamydosporia. Ann. Appl. Biol. 155:143-152.

6. Bonants, P. J. M., Flitters, P. F. L., Thijs, H., den Belder, E., Waalwijk, C., and Henfling, J. W. D. M. 1995. A basic serine protease from Paecilomyces lilacinus with biological activity against Meloidogyne hapla eggs. Microbiology 141:775-784.

7. Bourne, J. M., and Kerry, B. R. 1999. Effect of the host on the efficacy of Verticillium chlamydosporium as a biological control agent of root-knot nematodes at different nematode densities and fungal application rates. Soil Biol. Biochem. 31:75-84.

8. Bridge, J., and Page, S. L. J. 1980. Estimation of root-knot nematode infestation levels on roots using a rating chart. Trop. Pest Manage. 26:296-298.

9. Castrillo, L. A., Griggs, M. H., and Vandenberg, J. D. 2008. Quantitative detection of Beauveria bassiana GHA (Ascomycota: Hypocreales), a potential microbial control agent of the emerald ash borer, by use of realtime PCR. Biol. Control 45:163-169.
10. Ciancio, A., Loffredo, A., Paradies, F., Turtoro, C., and Finetti Sialer, M. 2005. Detection of Meloidogyne incognita and Pochonia chlamydosporia by fluorogenic molecular probes. Bull. OEPP/EPPO Bull. 35:157-164.

11. Evans, A. A. F., and Perry, R. N. 2009. Survival mechanisms. Pages 201221 in: Root-Knot Nematodes. R. N. Perry, M. Moens, and J. L. Starr, eds. CAB International, Wallingford, UK.

12. Greco, N., and De Vito, M. 2009. Population dynamics and damage levels. Pages 246-274 in : Root-Knot Nematodes. R. N. Perry, M. Moens, and J. L. Starr, eds. CAB International, Wallingford, UK.

13. Hirsch, P. R., Mauchline, T. H., Mendum, T. A., and Kerry, B. R. 2000. Detection of the nematophagous fungus Verticillium chlamydosporium in nematode-infested plant roots using PCR. Mycol. Res. 104:435-439.

14. Holland, R. J., Williams, K. L., and Khan, A. 1999. Infection of Meloidogyne javanica by Paecilomyces lilacinus. Nematology 1:131-139.

15. Hussey, R. S., and Barker, K. R. 1973. A comparison of methods collecting inocula of Meloidogyne spp. including a new technique. Plant Dis. Rep. 57:1025-1028.

16. Hussey, R. S., and Janssen, G. J. W. 2002. Root-knot nematodes: Meloidogyne species. Pages 43-70 in: Plant Resistance to Parasitic Nematodes. J. L. Starr, R. Cook, and J. Bridge, eds. CAB International, Wallingford, UK.

17. Jensen B., Knudsen, I. M. B., Madsen, M., and Jensen, D. F. 2004. Biopriming of infected carrot seed with an antagonist, Clonostachys rosea, selected for control of seedborne Alternaria spp. Phytopathology 94:551-560.

18. Kerry, B. R. 2001. Exploitation of the nematophagous fungal Verticillium chlamydosporium for the biological control of root-knot nematodes (Meloidogyne spp.). Pages 155-167 in: Fungi as Biocontrol Agents. T. M. Butt, C. Jackson, and N. Magan, eds. CAB International, Wallingford, UK.

19. Kerry, B. R., and Hidalgo-Diaz, L. 2004. Application of Pochonia chlamydosporia in the integrated control of root-knot nematodes on organically grown vegetable crops in Cuba. Pages 123-126 in: Multitrophic Interactions in Soil. R. A. Sikora, S. Gowen, R. Hauschild, and S. Kiewnick, eds. IOBC/wprs Bull. 27.

20. Khan, A., Williams, K. L., Molloy, M. P., and Nevalainen, H. K. M. 2003. Purification and characterization of a serine protease and chitinases from Paecilomyces lilacinus and detection of chitinase activity on 2D gels. Protein Expres. Purif. 32:210-220.

21. Khan, A., Williams, K. L., and Nevalainen, H. K. M. 2003. Testing the nematophagous biological control strain Paecilomyces lilacinus 251 for paecilotoxin production. FEMS Microbiol. Lett. 227:107-111.

22. Kiewnick, S. 2006. Effect of temperature on growth, germination, germtube extension and survival of Paecilomyces lilacinus strain 251. Biocontrol Sci. Technol. 16:535-546.

23. Kiewnick, S. 2006. Multitrophic interactions of Paecilomyces lilacinus strain 251 in the rhizosphere of host and non-host plants. Pages 53-61 in: Multitrophic Interactions in Soil. J. M. Raaijmakers and R. A. Sikora, eds. IOBC/wprs Bull. 29.

24. Kiewnick, S. 2007. Practicalities of developing and registering microbial biological control agents. CAB Rev. 2:1-11.

25. Kiewnick, S. 2009. Importance of multitrophic interactions for successful biocontrol of plant parasitic nematodes with Paecilomyces lilacinus strain 251. Pages 81-92 in: Plant Pathology in the 21st Century, Volume 1, Recent Developments in Management of Plant Diseases. U. Gisi, I. Chet, and M. L. Gullino, eds. Springer, The Netherlands.

26. Kiewnick, S., Lauritzen, A. J., and Eilenberg, J. 2006. Investigations on the colonization of root-knot nematode galls and egg masses by the antagonistic fungus Paecilomyces lilacinus strain 251. (Abstr.) Phytopathology 96:S60.

27. Kiewnick, S., and Sikora, R. A. 2006. Biological control of the root-knot nematode Meloidogyne incognita by Paecilomyces lilacinus strain 251. Biol. Control 38:179-187.

28. Kiewnick, S., and Sikora, R. A. 2006. Evaluation of Paecilomyces lilacinus strain 251 for the biological control of the northern root-knot nematode Meloidogyne hapla Chitwood. Nematology 8:69-78.

29. Kok, C. J., Papert, A., and Hok-A-Hin, C. H. 2001. Microflora of Meloidogyne egg masses: species composition, population density and effect on the biocontrol agent Verticillium chlamydosporium (Goddard). Nematology 3:729-734.

30. Larkin, R. P., and Fravel, D. R. 1999. Mechanisms of action and doseresponse relationships governing biological control of Fusarium wilt of tomato by non-pathogenic Fusarium spp. Phytopathology 89:1152-1161.

31. Mauchline, T. H., Kerry, B. R., and Hirsch, P. R. 2004. The biocontrol fungus Pochonia chlamydosporia shows nematodes host preference at the infraspecific level. Mycol. Res. 108:161-169.

32. McSpadden Gardener, B. 2006. Statistical analyses of microbiological and environmental data. Pages 555-585 in: Modern Soil Microbiology, 2nd ed. J. D. van Elsas and J. Jannson, eds. CRC Press, Boca Raton, FL.

33. Meyer, S. L. F., and Roberts, D. P. 2002. Combinations of biocontrol 
agents for management of plant-parasitic nematodes and soilborne plantpathogenic fungi. J. Nematol. 34:1-8.

34. Montesinos, E., and Bonaterra, A. 1996. Dose-response models in biological control of plant pathogens: An empirical verification. Phytopathology 86:464-472.

35. Morton, C. O., Hirsch, P. R., and Kerry, B. R. 2004. Infection of plantparasitic nematodes by nematophagous fungi-a review of the application of molecular biology to understand infection processes and to improve biological control. Nematology 6:161-170.

36. Orion, D., Kritzman, G., Meyer, S. L. F., Erbe, E. F., and Chitwood, D. J. 2001. A role of the gelatinous matrix in the resistance of root-knot nematode (Meloidogyne spp.) eggs to microorganisms. J. Nematol. 33:203-207.

37. Pantelides, I. S., Tjamos, S. E., Striglis, I. A., Chatzpavlidis, I., and Paplomatas, E. J. 2009. Mode of action of a non-pathogenic Fusarium oxysporum strain against Verticillium dahliae using real time QPCR analysis and biomarker transformation. Biol. Control 50:30-36.

38. Rumbos, C., and Kiewnick, S. 2006. Effect of plant species on persistence of Paecilomyces lilacinus strain 251 and on root colonization by the fungus. Plant Soil 28:25-31.

39. Rumbos, C., Mendoza, A., Sikora, R A., and Kiewnick, S. 2008. Persistence of the nematophagous fungus Paecilomyces lilacinus strain 251 in soil under controlled conditions. Biocontrol Sci. Technol. Vol. 18:1041-1050.

40. Sharon, E., Chet, I., Viterbo, A., Bar-Eyal, M., Nagan, H., Samuels, G. J., and Spiegel, Y. 2007. Parasitism of Trichoderma on Meloidogyne javanica and role of the gelatinous matrix. Eur. J. Plant. Pathol. 118:247-258.

41. Takaneka, S., Sekiguchi, H., Kazuhiro, N., Tojo, M., Masunaka, A., and Takahashi, H. 2008. Colonization of Pythium oligandrum in the tomato rhizosphere for biological control of the bacterial wilt disease analyzed by real-time PCR and confocal laser-scanning microscopy. Phytopathology 98:187-195.

42. Yao, Y., Nellaker, C., and Karlsson, H. 2006. Evaluation of minor groove binding probe and TaqMan probe PCR assays: influence of mismatches and template complexity on quantification. Mol. Cell. Probes 20:311-316.

43. Zar, H. J. 1999. Biostatistical Analysis. Prentice-Hall Inc., Upper Saddle River, NJ. 